\title{
Le Théâtre congolais
}

Au théâtre du parc de Bock, à Léopoldville, a été présentée une pièce composée en lingala par le peintre congolais Albert Mongita. Elle fut jouée sous les auspices du 'Cercle d'étude et d'agrément' par une quarantaine d'acteurs et $d$ ' actrices noirs, presque tous des amateurs.

Cette pièce, dont seul le canevas a été écrit, est une histoire fort amusante qui brusquement tourne au drame. Elle se déroule dans la brousse à la fin du siècle dernier. Un père convoque les prétendants de sa fille. Devant le village rassemblé chacun des jeunes gens vient vanter ses qualités jusqu'à ce que le choix de la jeune fille se porte sur l'un d'eux: Mandjodjo. L'élu ramène sa femme chez lui mais bientôt la mésentente surgit, le mari ne voulant pas partager avec elle le produit de sa chasse. Mais la jeune épouse est rusée. Elle s'en va à la pèche et à son tour refuse de partager les poissons avec son mari. Celui-ci use alors d'un subterfuge et, sous le déguisement d'un vieillard, vient demander du poisson. Mais la femme n'est pas dupée et une nouvelle fois refuse. Le conflit s'envenime, Mandjodjo en colère frappe sa femme d'un coup de couteau. La famille de la victime est alertée et le père provoque son gendre en combat singulier. A ce moment, et fort à propos, Stanley survient qui rétablit l'ordre, expédie le mari en prison et guérit l'épouse blessée.

Les acteurs n'ont reçu que des directives plus ou moins précises sur le caractère du personnage qu'ils représentent. Pour le reste, ils brodent librement sur le canevas en suivant l'inspiration du moment. L'habitude des palabres, si répandue parmi les Congolais, confère aux acteurs une exceptionnelle facilité d'improvisation et d'éloquence.

Ce spectacle où alternent danses, chants, et dialogues, est typiquement populaire, c'est-àdire accessible à un public très large. Il présente des aspects de la vie coutumière telle qu'elle apparaît encore en brousse. Il décrit de nombreux petits faits familiers comme les spectateurs en vivent tous les jours. La psychologie des personnages est rudimentaire mais caricaturée avec beaucoup de justesse; les discussions surgissant entre eux sont étonnantes de naturel.

Ce jeune théâtre présente cependant des points faibles. Le talent d'improvisation des acteurs ainsi que les rebondissements de l'intrigue entraînent des longueurs ressenties surtout par le public européen mais qui n'échappent pas non plus aux Congolais.

L'accompagnement de tam-tam, les danses et les chants indigènes ont largement contribué à créer un climat évocateur pour ce spectacle qui s'est déroulé dans un décor simplifié représentant une case dans la forêt. Les acteurs avaient revêtu des peaux de bête et des pagnes de raphia.

La pièce de Mongita, comme plusieurs essais qui la précédèrent, prouve qu'il est possible de créer un théâtre où l'inspiration congolaise occuperait la part essentielle.

C'est, d'ailleurs, en accentuant encore ce retour aux sources congolaises que le théâtre indigène pourra devenir une réalité vivante en répondant alors à un réel besoin d'expression de l'âme congolaise.

\section{Mission au Tchad et dans le Cameroun Septentrional}

Cetre mission qui a reçu l'appui du Centre National de la Recherche Scientifique, du Gouvernement du Tchad et de l'Académie des Sciences Coloniales (Prix Georges Bruel) se compose de Jean-Paul Lebeuf et d'Annie Masson Detourbet. Son séjour prévu est de six à sept mois (décembre 1951-juin 1952).

Elle a pour but la poursuite de l'étude systématique de la région du Tchad (A.E.F.) et du Nord-Cameroun commencée en 1936 par la Mission Sahara-Cameroun ( $4^{\mathrm{e}}$ Mission Griaule) et continuée par les différentes missions dirigées par J-P. Lebeuf qui faisait partie de la Mission Sahara-Cameroun.

Au Tchad, outre l'exploitation archéologique de la butte sao de Midigué, la mission continuera à étudier les Kotoko, descendants des Sao, chez lesquels une attention particulière sera 